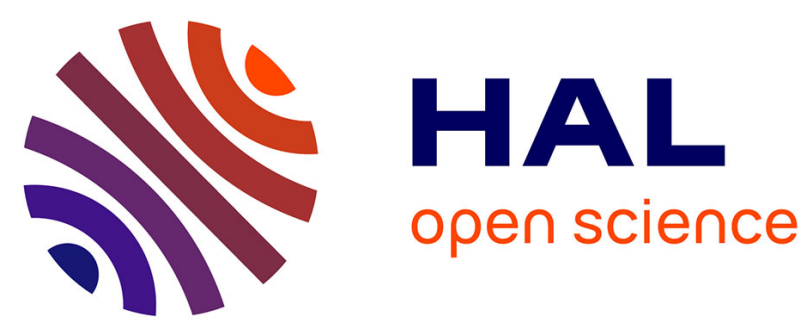

\title{
Improving Understanding of Long-Term Cardiac Functional Remodelling via Cross-Sectional Analysis of Polyaffine Motion Parameters
}

Kristin Mcleod, Maxime Sermesant, Xavier Pennec

\section{To cite this version:}

Kristin Mcleod, Maxime Sermesant, Xavier Pennec. Improving Understanding of Long-Term Cardiac Functional Remodelling via Cross-Sectional Analysis of Polyaffine Motion Parameters. FIMH 2017 9th International Conference on Functional Imaging and Modeling of the Heart, Jun 2017, Toronto, Canada. pp.51 - 59, 10.1007/978-3-319-59448-4_6 . hal-01574837

\section{HAL Id: hal-01574837 \\ https://hal.inria.fr/hal-01574837}

Submitted on 16 Aug 2017

HAL is a multi-disciplinary open access archive for the deposit and dissemination of scientific research documents, whether they are published or not. The documents may come from teaching and research institutions in France or abroad, or from public or private research centers.
L'archive ouverte pluridisciplinaire HAL, est destinée au dépôt et à la diffusion de documents scientifiques de niveau recherche, publiés ou non, émanant des établissements d'enseignement et de recherche français ou étrangers, des laboratoires publics ou privés. 


\title{
Improving Understanding of Long-Term Cardiac Functional Remodelling via Cross-Sectional Analysis of Polyaffine Motion Parameters
}

\author{
K. McLeod ${ }^{1,2}$, M. Sermesant ${ }^{3}$, and X. Pennec ${ }^{3}$ \\ 1 Centre for Cardiological Innovation, Simula Research Laboratory, Oslo, Norway \\ 2 KardioMe s.r.o, Slovakia \\ 3 Université Côte dAzur, Inria, France
}

\begin{abstract}
Changes in cardiac motion dynamics occur as a direct result of alterations in structure, hemodynamics, and electrical activation. Abnormal ventricular motion compromises long-term sustainability of heart function. While motion abnormalities are reasonably well documented and have been identified for many conditions, the remodelling process that occurs as a condition progresses is not well understood. Thanks to the recent development of a method to quantify full ventricular motion (as opposed to 1D abstractions of the motion) with few comparable parameters, population-based statistical analysis is possible. A method for describing functional remodelling is proposed by performing statistical cross-sectional analysis of spatio-temporally aligned subject-specific polyaffine motion parameters. The proposed method is applied to pathological and control datasets to compare functional remodelling occurring as a process of disease as opposed to a process of ageing.
\end{abstract}

\section{Introduction}

Cardiac function can be altered under disease states, reducing the long-term ability of the heart to pump efficiently. While there has been effort dedicated towards understanding typical functional abnormalities occurring for different diseases, the functional remodelling processes that occur over time are poorly understood. A good understanding of functional remodelling is key in optimising treatment to, for example, predict long-term functional remodelling for decision making of cardiac implants, or to determine the best timing to intervene for valve replacement surgery.

\subsection{State-of-the-art in Cardiac Motion Tracking}

Cardiac motion tracking via non-rigid registration has been used in recent years to quantify 3D motion of the heart. Quantitative measures of motion can be used to detect abnormal regions and to provide more detailed measures beyond simple abstractions of function such as volumes or ejection fraction. An overview of recent methods is given in [1]. Population-based analysis of motion has been 
used to quantify changes over time in cardiac function by performing groupwise analysis of strain derived from tagged magnetic resonance imaging [2] or by studying spatio-temporally aligned displacements [3]. While informative, these methods only give a snapshot into the function of the heart and do not provide insight into how the function remodels over time.

\subsection{State-of-the-art in Cardiac Remodelling}

Functional changes have been studied by, for example, comparing strain values before and shortly after myocardial infarction [4], and pre- and post-surgery for aortic stenosis patients [5]. However, few studies have investigated long-term functional changes, even for 1D measures of strain [6]. Analysis of changes in the heart over time is an ongoing area of research, which has traditionally been addressed using longitudinal patient data. In light of the fact that longitudinal studies require long-term follow-up of the same patients, potentially over years, the prospect of using the results of such studies to guide therapy planning remains low. In contrast, cross-sectional analysis of patients within the same population has the ability to describe long-term changes without the need to wait years for a patient to age. Long-term cardiac structural remodelling was analysed by Mansi et al. [7] to study the long-term shape remodelling that occurs in the right ventricle of Tetralogy of Fallot patients in response to increased load caused by regurgitated blood from the lungs. This study was performed only on the end diastolic phase of the cardiac cycle, and is therefore not able to capture functional remodelling that (most likely) occurs over time in these patients.

\subsection{Aim and Paper Organisation}

In the present work we propose to analyse long-term remodelling of cardiac function using a method that is able to represent the full 3D dynamics of the heart. The proposed method makes use of a recent method to track cardiac motion by describing motion regionally as affine transformations, and fusing these to a global smooth transformation [8]. In contrast to previous work, where the motion was analysed only at a single time point, the present work builds upon recent effort in cardiac motion tracking and population-based analysis to analyse changes in motion over time. Here, we are interested in studying motion over two time scales: the 3D dynamics during the heart beat (motion parameters), and the remodelling over years (changes in these motion parameters). The contributions of the present work are:

1. Derivation of a long-term functional model using image registration, polyaffine projection, and cross-sectional statistical analysis

2. Quantitative analysis of the accuracy of predicting age given motion parameters using leave-one-out numerical validation

3. Qualitative analysis of the accuracy of predicting motion given age by analysing motion sequences and image-derived parameters 
The remainder of the paper is organised as follows: Sec 2 summarises the techniques used to track the motion of the left ventricle and project this onto a polyaffine space, in which the polyaffine motion parameters can be analysed. Sec. 3 describes the proposed method to perform cross-sectional analysis of the polyaffine motion parameters. Sec. 4 describes experiments performed to quantitatively and qualitatively evaluate the proposed method, and in Sec. 5 the methods are evaluated, discussed, and future perspectives are given.

\section{Background: Polyaffine Motion Tracking}

A global (affine) description of motion is not sufficient to characterise the motion of the heart. On the other hand, voxel-wise descriptors most likely over-represent the motion when considering that the heart consists of connected tissue which moves in an elastic manner. Describing the motion at a regional level can provide a suitable compromise between these two, to sufficiently describe the motion while representing the motion with as few parameters as possible. Polyaffine projection allows motion to be described at a regional level while maintaining a globally smooth transformation by means of weight functions that smooth the transformation between regions. Moreover, for the purpose of estimating the remodelling over time, it is important to have a robust yet informative description of the motion. We believe that the Polyaffine transformations provide a good trade-off.

Polyaffine projection from a dense deformation field was proposed by Seiler et al. [9] via $\log$-affine matrices $M_{i}$ described in each region $i$ that are fused to a global deformation field by $v(x)=\sum_{i}^{N} \omega_{i}(x) M_{i} x . \omega(x)$ are weight parameters in the $N$ regions, describing the weight of the transformation of each region of a given voxel $x$ written in homogeneous coordinates. As described in [9], the polyaffine projection can be formulated as a linear least squares problem by solving $\nabla C_{M}=0$, where $C$ is described by the following formula:

$$
C(M)=\int_{\Pi}\left\|\sum_{i} \omega_{i}(x) \cdot M_{i} \tilde{x}-\boldsymbol{v}(x)\right\|^{2} d x .
$$

The polyaffine projection technique used in this work was implemented following the method described in [8], which was developed specifically for motion tracking of the left ventricle of the heart.

\section{Methods: Regression of Polyaffine Motion Parameters}

The compact parameterisation of motion parameters using the polyaffine formulation described in the previous section enables cross-sectional analysis of motion. Common methods for cross-sectional analysis include principal component analysis (PCA) and partial least squares (PLS). PLS has the advantage over PCA of computing directly the components most related to the output 
variable. In both cases, the regression model is performed by computing the motion parameters from age since the converse requires prediction of many output parameters: motion described by 5916 parameters per subject (12 affine parameters $\times 17$ regions $\times 29$ time frames), given few input parameters: age described by a single value (in years). Following the method for computing shape features from age proposed by Mansi et al. [7], canonical correlation analysis (CCA) can be used to reverse the relationship in order to obtain a model of motion given age. The proposed pipeline is summarised in Fig. 1.

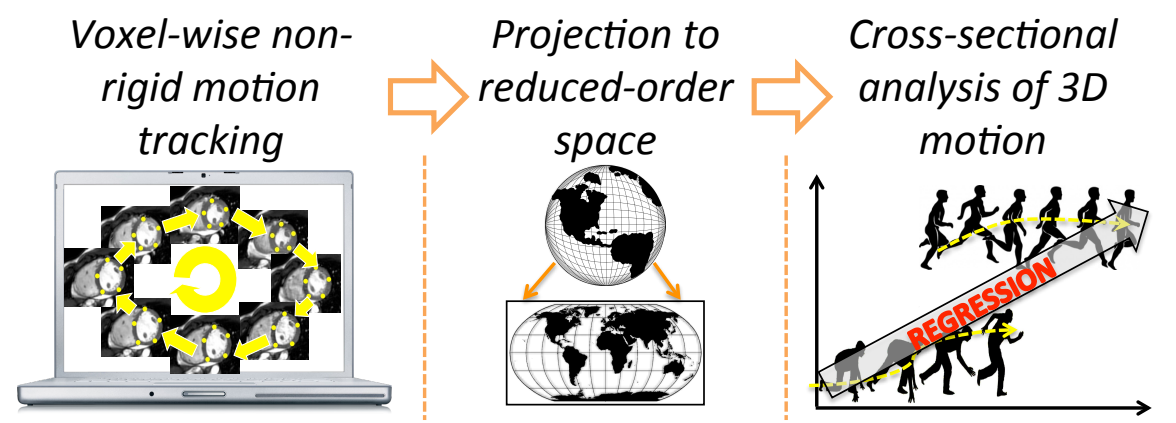

Fig. 1: Proposed pipeline from image to motion model. Non-rigid motion tracking is performed to describe the voxel-wise motion in the heart. This is then projected to a polyaffine space in order to represent the motion with few parameters, consistently defined from one subject to another. Finally, cross-sectional analysis is performed on the motion parameters to formulate a generative model of functional remodelling.

\section{Experiments: Polyaffine Motion Parameter Regression}

Two types of experiments were performed to validate the proposed method. The first experiment was a quantitative validation of the accuracy of predicting age given the motion parameters. While this is not a clinically meaningful validation (since the age of patients is generally known), it serves as a means to validate the regression since it is straightforward to compute the accuracy when predicting a single output value. The second experiment was a qualitative assessment of the prediction of motion given age, which is difficult to validate quantitatively given the large number of predicted parameters, but can be analysed qualitatively by visualising the output to determine if it matches physiological motion dynamics.

\subsection{Testing Datasets}

The motion parameters used in the experiments were taken directly from the previous work of [8], which consists of two datasets; a healthy control dataset, 
and a pathological group of Tetralogy of Fallot patients. The healthy control subjects from the openly available STACOM 2011 cardiac motion tracking challenge dataset [1] $(n=15,3$ female, mean age $\pm \mathrm{SD}=28 \pm 5)$ were used since these have already been used to validate the polyaffine cardiac motion tracking. The pathological subjects were a dataset of Tetralogy of Fallot patients $(n=10$, 5 female, mean age $\pm \mathrm{SD}=21 \pm 7$ ), which were already used to analyse the polyaffine motion parameters in [10]. The Tetralogy of Fallot cohort forms a suitable testing set for this problem since there are known functional changes over time in these patients in response to poor pulmonary valve function or in the absence of a pulmonary valve. Furthermore, modelling functional remodelling in this population complements the previous analysis of shape remodelling performed by Mansi et al. [7]. In both cohorts, only left ventricular motion was tracked due to the absence of open testing sets to validate bi-ventricle motion tracking methods. The motion tracking results from these experiments were consistent with those presented in [8].

\subsection{Statistical Analysis}

Following the techniques described in [7] to predict shape remodelling, partial least squares (PLS) was applied to each vectorised motion array to reduce the parameter space to a vector of PLS component loadings, from which CCA between the loading vector (representing motion) and age was computed. CCA was performed iteratively on the number of components, to find the minimal number of components required to obtain a statistically significant combination of PLS components with respect to the dependent variable. Statistical significance was computed using the Bartlett-Lawley hypothesis test. Since we were interested in modelling motion (and not age), the important variable to explain is $Y$ (vector of Polyaffine motion parameters from $M$ ), rather than $X$ (age). PLS captured more than $90 \%$ of the variability in $Y$ with only 2 components for the ToF group, and 7 components for the control group.

The canonical correlations are plotted for each component from PLS of each group in Fig. 2. The canonical correlations for PLS are ordered in magnitude by design since PLS is maximising the variance in $Y$ and covariance in $X$ and $Y$.

Quantitative Validation of the Prediction of Age Given Motion The testing error of predicting age given motion was computed by performing leaveone-out validation (training on $n-1$ subjects, and predicting the age of the $n^{\text {th }}$ subject. In all error computations, the number of CCA components for the full training set were used. The training error of the Tetralogy of Fallot group was 0.30 years and the testing error was 5.85 years. For the control group, the respective training and testing errors were 0.47 years and 6.81 years. For the ToF group, the yielded errors were below the population standard deviation of age (6.49). For the control group, however, the testing errors were above the population standard deviation of 5.0 years. 

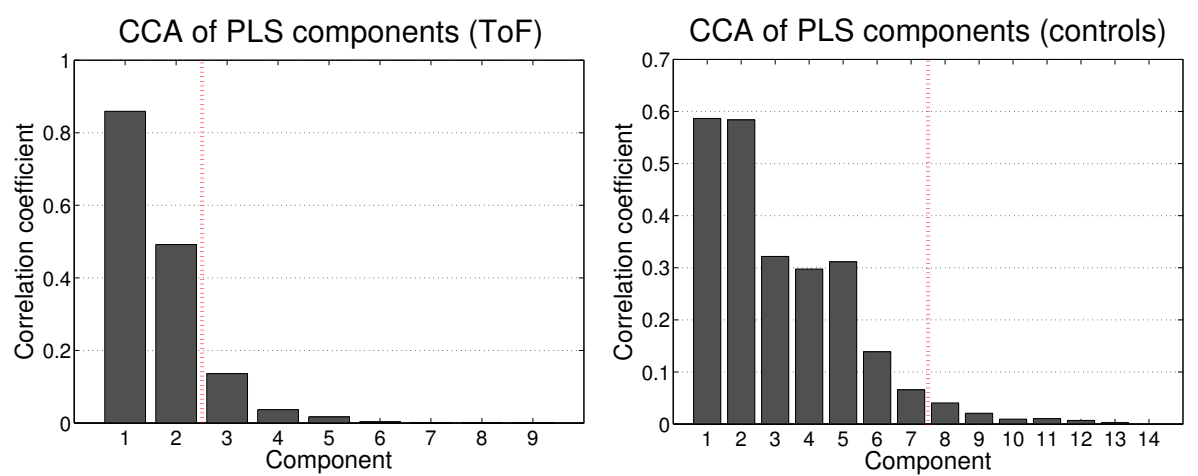

Fig. 2: Canonical correlations for PLS for the Tetralogy of Fallot group (left) and the control group (right). The red line indicates the minimum number of components needed for each group to obtain statistically significant combinations of the modes. Fewer components were required in the more heterogeneous pathological group (i.e. the ToF group) than relatively homogeneous control group.

Qualitative Analysis of Prediction of Motion Given Age Quantitative validation of the motion parameters computed from age would require longitudinal data, which is a challenge to obtain given the long time intervals required between time points. Furthermore, quantitative validation voxel-wise is challenging due to the fact that the heart remodels structurally over time in addition to the functional remodelling. Since there is currently no method to identify how/where voxels move over time as a result of structural remodelling compared to those that move as a result of functional remodelling, quantitative validation of the motion estimation is not possible. Therefore, only qualitative analysis of motion via visualisation of the motion sequences was performed. Fig. 3 shows the predictive (healthy or pathological) deformation models applied to a single magnetic resonance image using the motion visualisation method described in [8], shown here at two phases of the cardiac cycle to compare the remodelling of motion in the Tetralogy of Fallot group to the control group. As expected, the control group shows little changes in motion through ageing, since the population is healthy the motion should remain stable over time. In contrast, the Tetralogy of Fallot group show large differences in motion over time, particularly in the free wall.

As a preliminary means of quantitative validation, the predicted motion sequences for the Tetralogy of Fallot group were segmented to obtain the left ventricular volumes, plotted in Fig. 4. As the curves show, the minimum of the curves increase with age, which ultimately decreases ejection fraction; a commonly used measure of function by cardiologists. Ejection fraction is known to decrease with age after initial repair in these patients [11]. 

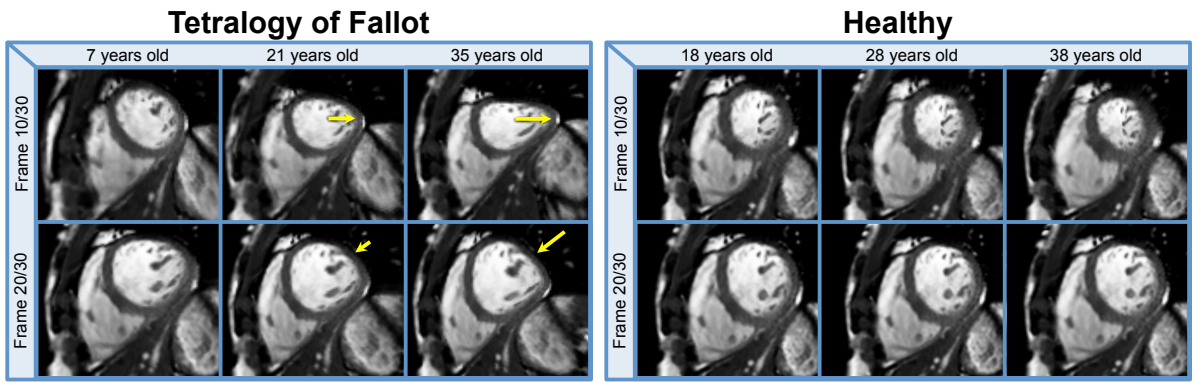

Fig. 3: Snapshots of the sequences shown at two phases of the cardiac cycle: frame 10 (top row) and frame 20 (bottom row) of a total of 30 frames for the Tetralogy of Fallot group (left) and healthy control group (right). Three ages are shown for each group at -2 standard deviations of the age, the mean age, and +2 standard deviations of age. Note that the motion in the control group remains stable over the ages, since very little motion changes occur over normal ageing. In contrast, noticeable changes in the motion of the Tetralogy of Fallot group are visible, as highlighted by the yellow arrows.

\section{Discussion and Perspectives}

The experiments suggest that the proposed methods are promising for computing the long-term functional changes in the left ventricle. The computation of age from motion parameters was more accurate in the Tetralogy of Fallot group than the healthy control group, which is as expected since the control group consists of a range of ages in which the motion parameters should be reasonably stable (thus making regression challenging). The qualitative validation suggests that the methods are able to predict feasible motion dynamics from age alone, despite the small sizes of the datasets. These findings should be, however, confirmed in a larger population in which longitudinal data is available to quantitatively validate the prediction of motion from age. Additionally, with larger datasets the models could be sub-grouped to ensure that the change due to the evolution is much larger than the cross-sectional variability.

An important limitation of the present work is that no longitudinal data was used for the regression. As mentioned by Eng et al. [12], cross-sectional analysis can underestimate size changes over time compared to longitudinal analysis. Since longitudinal data was not available at the time of this study they were not used, therefore future studies will focus on a combined longitudinal and cross-sectional study design.

This work presents only functional changes without consideration of structure, despite the fact that these are inherently coupled and could be more informative when used in combination. A very interesting future work will address coupling shape (for example using the methods in [7]) with function.

In the present work, regression was performed on matricised tensors. However, the regression could be performed directly on the tensors. Performing re- 


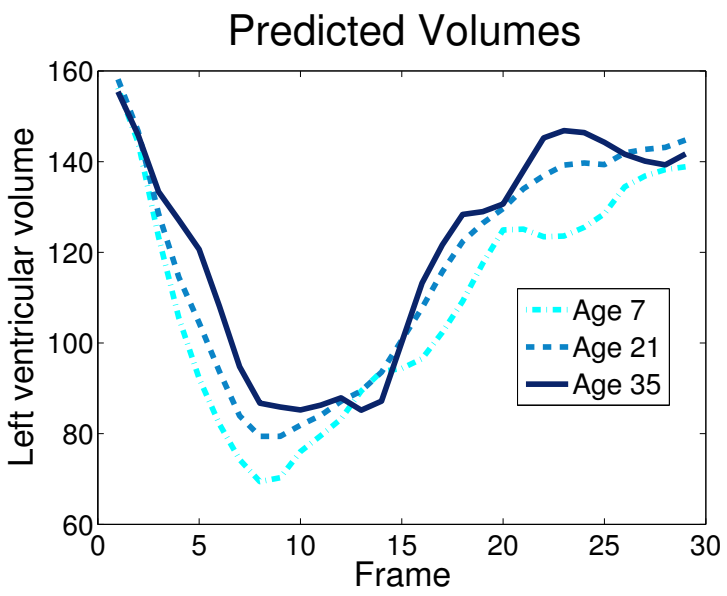

Fig. 4: Segmented volumes computed from sequences predicted from the Tetralogy of Fallot group model. Ejection fraction (used as a measure of cardiac function) decreased over time, consistent with clinical findings in this patient group.

gression directly on the tensor may be an advantage for retaining the structure of the tensor in the regression, and to reduce the number of parameters required to describe the generative motion models.

\section{Conclusions}

In this work we propose a method to analyse motion changes in a population over time using a non-rigid registration algorithm in conjunction with polyaffine projection and statistical analysis. The proposed method was validated quantitatively and qualitatively on a control dataset and on a dataset of Tetralogy of Fallot patients and complements the work of [7] to allow future potential work on joint shape and motion evolution modelling. This is, to our knowledge, the first method that is able to predict long-term functional remodelling at a level that retains the full complexity of motion dynamics.

Acknowledgements: This project was carried out as a part of the Centre for Cardiological Innovation (CCI), Norway, funded by the Research Council of Norway. The authors would like to thank Tommaso Mansi for providing the code for computing CCA.

\section{References}

1. Tobon-Gomez, C., De-Craene, M., et al: Benchmarking framework for myocardial tracking and deformation algorithms: An open access database. Med. Im. Anal. (2013)

2. Qian, Z., Liu, Q., Metaxas, D.N., Axel, L.: Identifying regional cardiac abnormalities from myocardial strains using nontracking-based strain estimation and spatio-temporal tensor analysis. IEEE Trans. Med. Im. 30(12) (2011) 2017-2029 
3. Rougon, N.F., Petitjean, C., Preteux, F.J.: Building and using a statistical 3D motion atlas for analyzing myocardial contraction in MRI. In: Proc. SPIE 5370, Medical Imaging 2004: Image Processing. (2004) 253-264

4. Gerber, B.L., Rochitte, C.E., Melin, J.A., McVeigh, E.R., Bluemke, D.A., Wu, K.C., Becker, L.C., Lima, J.A.: Microvascular obstruction and left ventricular remodeling early after acute myocardial infarction. Circulation 101(23) (2000)

5. Bauer, F., Eltchaninoff, H., Tron, C., Lesault, P.F., Agatiello, C., Nercolini, D., Derumeaux, G., Cribier, A.: Acute improvement in global and regional left ventricular systolic function after percutaneous heart valve implantation in patients with symptomatic aortic stenosis. Circulation 110(11) (2004) 1473-1476

6. Kuznetsova, T., Thijs, L., Knez, J., Cauwenberghs, N., Petit, T., Gu, Y.M., Zhang, Z., Staessen, J.A.: Longitudinal changes in left ventricular diastolic function in a general population. Circulation: Cardiovascular Imaging 8(4) (2015)

7. Mansi, T., Voigt, I., Leonardi, B., Pennec, X., Durrleman, S., Sermesant, M., Delingette, H., Taylor, A.M., Boudjemline, Y., Pongiglione, G., Ayache, N.: A statistical model for quantification and prediction of cardiac remodelling: Application to tetralogy of fallot. IEEE Trans. Med. Im. 9(30) (2011) 1605-1616

8. McLeod, K., Sermesant, M., Beerbaum, P., Pennec, X.: Spatio-temporal tensor decomposition of a polyaffine motion model for a better analysis of pathological left ventricular dynamics. IEEE Trans. Med. Im. (2015)

9. Seiler, C., Pennec, X., Reyes, M.: Capturing the multiscale anatomical shape variability with polyaffine transformation trees. Med. Image Anal. (2012)

10. Mcleod, K., Sermesant, M., Beerbaum, P., Pennec, X.: Descriptive and intuitive population-based cardiac motion analysis via sparsity constrained tensor decomposition. In: Medical Image Computing and Computer-Assisted InterventionMICCAI 2015. Springer (2015) 419-426

11. Waien, S.A., Liu, P.P., Ross, B.L., Williams, W.G., Webb, G.D., Mclaughlin, P.R.: Serial follow-up of adults with repaired tetralogy of fallot. Journal of the American College of Cardiology 20(2) (1992) 295-300

12. Eng, J., McClelland, R.L., Gomes, A.S., Hundley, W.G., Cheng, S., Wu, C.O., Carr, J.J., Shea, S., Bluemke, D.A., Lima, J.A.: Adverse left ventricular remodeling and age assessed with cardiac $\mathrm{mr}$ imaging: the multi-ethnic study of atherosclerosis. Radiology 278(3) (2015) 714-722 